\title{
Where Chemistry Meets Art: An Exploration of Nano World through Mathematical Beading ${ }^{\dagger}$
}

\author{
Bih-Yaw Jin ${ }^{1, *}$ and Chia-Chin Tsoo ${ }^{2}$ \\ 1 Department of Chemistry, National Taiwan University, Taipei 10617, Taiwan \\ 2 National Center for High-Performance Computing, Hsinchu 30076, Taiwan; chchts@gmail.com \\ * Correspondence: byjin@ntu.edu.tw \\ † Presented at Symmetry 2017-The First International Conference on Symmetry, Barcelona, Spain, \\ 16-18 October 2017.
}

Published: 5 January 2018

Polyhedra excite the imagination not only of mathematicians, engineers, architects, and artists, but also of biologists and chemists. "Molecular modelling is a constitutive, yet overlooked, element of the practice of chemistry" [1]. A case in point is C60 or Buckyball, where paper models played a pivotal role in elucidating the truncated icosahedral shape of C60, for which Kroto, Curl, and Smalley were awarded the 1996 Nobel Prize in Chemistry. Unfortunately, some molecular structures, including fullerenes, are inaccessible with standard molecular modeling kits due, in part, to the large number of atoms involved. In this poster, a simple method to construct molecular models for arbitrary fullerenes, nanotori, high-genus fullerenes, helically coiled carbon nantotubes, carbon torus knots, and carbon Schwarzites using beads is described [2-4]. In a short amount of time, students of all ages can create a fullerene museum on their desk. Bead models of C60 and C70 constructed from 90 and 105 beads and single nylon fishing threads are shown in the following figure, respectively. In an over-simplified view, bead models of fullerenes represent the electron-pair domain model for trivalent systems where each bead represents the electron density of a carbon-carbon bond in the fullerene. Owing to the similarity between microscopic valence shell electron pair repulsion and macroscopic mechanical hard-sphere interactions in the trivalent beaded models, the shape of a bead model is similar to the true molecular structure of the corresponding fullerene. In addition to trivalent graphitic structures, other successful applications of mathematical beading to molecular physical modeling include valence sphere models of simple organic molecules, extended metal atom chains, polyhedral models of inorganic structures, Mackay's polyhedra, diamond, lonsdaleite, and various tetravalent zeolites.

\section{References}

1. Francoeur, E. The forgotten tool: The design and use of molecular models. Soc. Stud. Sci. 1997, 27, 7-40.

2. Jin, B.-Y.; Chuang, C.; Tsoo, C.-C. Construction of Physical Models for Arbitrary Fullerenes with Beads: Realization of Tangent-Sphere Model. J. Chin. Chem. Soc. 2010, 57, 316.

3. Jin, B.-Y.; Chuang, C.; Tsoo, C.-C. Constructing Molecules with Beads: The Geometry of Topologically Nontrivial Fullerenes. In Proceedings of Bridges: Mathematical Connections in Art, Music, and Science, Pécs, Hungary, 24-29 July 2010; pp. 391-394.

4. Chuang, C.; Jin, B.-Y.; Tsoo, C.-C.; Tang, N.Y.-W.; Cheung, M.P.S.; Cuccia, L.A. Molecular Modeling of Fullerenes with Beads. J. Chem. Edu. 2012, 89, 414-416. 